\title{
Pressure effects on $\mathrm{NaMnF}_{4}$ : Structural correlations and Jahn-Teller effect from crystal-field spectroscopy
}

\author{
F. Aguado, ${ }^{1}$ F. Rodríguez, ${ }^{1, *}$ and P. Núñez ${ }^{2}$ \\ ${ }^{I}$ DCITIMAC, Facultad de Ciencias, Universidad de Cantabria, Santander 39005, Spain \\ ${ }^{2}$ Departamento de Química Inorgánica, Universidad de la Laguna, Tenerife 38200, Spain
}

(Received 18 October 2002; revised manuscript received 23 December 2002; published 12 May 2003)

\begin{abstract}
This work investigates the optical absorption spectrum of the $\mathrm{NaMnF}_{4}$-layered perovskite and its variation with pressure. The spectrum basically consists of three broadbands located at 1.916, 2.263, and $2.817 \mathrm{eV}$, which correspond to the crystal-field (CF) transitions ${ }^{5} B_{1 g} \rightarrow{ }^{5} \Gamma_{i}\left(\Gamma_{i}=A_{1 g}, B_{2 g}\right.$, and $\left.E_{g}\right)$ with the Jahn-Teller(JT-) distorted $\mathrm{MnF}_{6}^{3-}$ complex $\left(\mathrm{Mn}^{3+} d^{4}\right.$ configuration). In addition, there are two spin-flip ${ }^{5} B_{1 g} \rightarrow{ }^{3} B_{1 g}$ peaks at 2.397 and $2.890 \mathrm{eV}$, which are activated by the exchange mechanism. Their variation with pressure reveals that the JT energy does not change significantly with pressure: $\partial E_{\mathrm{JT}} / \partial P=0.8 \mathrm{meV} / \mathrm{GPa}$. Furthermore, the variation of the JT tetragonal splitting of the parent octahedral $e_{g}$ and $t_{2 g}$, termed $\Delta_{e}$ and $\Delta_{t}$, respectively, clearly indicate that $\partial \Delta_{e} / \partial P \ll \partial \Delta_{t} / \partial P$, although $\Delta_{e} \approx 4 \Delta_{t}$. The CF energies and their pressure shift are explained in terms of local structural changes within the $\mathrm{MnF}_{6}^{3-}$ complex induced by pressure. The structural correlation analysis reveals that the reduction of the $\mathrm{MnF}_{6}^{3-} \mathrm{JT}$ distortion is smaller than the expected one on the basis of the crystal volume reduction, thus indicating tilt phenomena. This interpretation is supported by the decrease of in-layer Mn-F-Mn superexchange, such as is derived from the optical spectra. We demonstrate that the equatorial and axial distances decrease from 1.839 to $1.808 \AA$ and from 2.167 to $2.107 \AA$, respectively, in the $0-10 \mathrm{GPa}$ range.
\end{abstract}

DOI: 10.1103/PhysRevB.67.205101

PACS number(s): 71.70.Ch, 61.50.Ks, 71.70.Gm, 81.40.Vw

\section{INTRODUCTION}

$\mathrm{Mn}^{3+}$ compounds have received considerable attention due to the variety of structures and associated properties they present in different application fields: e.g., solid-state laser $\left(\mathrm{Mn}^{3+}\right.$-doped $\left.\mathrm{Y}_{3} \mathrm{Al}_{5} \mathrm{O}_{12}\right)$ (Refs. 1-3), dichroism $\left(\mathrm{Tl}_{2} \mathrm{MnF}_{5} \cdot \mathrm{H}_{2} \mathrm{O}\right)$ (Ref. 4), transparent ferromagnets $\left(\mathrm{CsMnF}_{4}\right)$ (Refs. 5-8), colossal magnetoresistance $\left(\mathrm{Sm}_{0.55} \mathrm{Sr}_{0.45} \mathrm{MnO}_{3}\right)$ (Refs. 9-11), or metal-insulator transition $\left(\mathrm{LaMnO}_{3}\right)($ Ref. 12) are examples of this ample behavior. Particularly, $\mathrm{Mn}^{3+}$ fluorides display a large variety of crystal structures, which are strongly related to the JahnTeller (JT) effect of $\left.\mathrm{Mn}^{3+}\left(3 d^{4}\right)\right)^{4,6,13-19}$ Depending on the ligand sharing of $\mathrm{MnF}_{6}^{3-}$ complexes, the crystal structure shows a different dimensional arrangement. It can be either zero dimensional (OD) if there is no ligand sharing (i.e., isolated $\mathrm{MnF}_{6}^{3-}$ units like in $\left.\mathrm{Na}_{3} \mathrm{MnF}_{6}\right),{ }^{20}$ 1D linear chains if they share the two axially ligands $\left(\mathrm{Na}_{2} \mathrm{MnF}_{5}\right){ }^{21}$ or $2 \mathrm{D}$ layers if four ligands are shared within the plane forming layers $\left(\mathrm{NaMnF}_{4}\right) .{ }^{22}$ The latter case, which corresponds to the title compound, displays an antiferrodistortive structure, in which the in-plane equatorial ligands of a given $\mathrm{MnF}_{6}^{3-}$ complex act as axial ligands of two nearest-neighbor complexes (Fig. 1). This situation favors a ferromagnetic Mn-F-Mn superexchange within the layer provided that the Mn-F-Mn tilt angle is not far from $180^{\circ} .5,6,23$ The present atomic arrangement simply explains the occurrence of ferromagnetism below $T_{C}=19 \mathrm{~K}$ in $\mathrm{CsMnF}_{4} .{ }^{5} \mathrm{~A}$ review on the magnetic properties of $\mathrm{Mn}^{3+}$ fluorides can be found elsewhere. ${ }^{6} \mathrm{MnF}_{6}^{3-}$ displays axially elongated coordination geometry along the $\mathrm{Mn}^{3+}$ compound series due to the JT effect. The associated distortion strongly depends on the crystal dimensionality: the higher the dimensionality, the greater the JT distortion. ${ }^{4}$ This structural correlation is important since the optical and magnetic properties rely not only on the superexchange Mn-F$\mathrm{Mn}$, but also on the JT distortion, which mainly determines the crystal-field (CF) electron structure. There has been interest in $\mathrm{Mn}^{3+}$ fluorides in the search for new ferromagnets as well as potential systems to induce structural changes associated with the high-spin to low-spin (HS-LS) electron ground-state crossover $\left[{ }^{5} E_{g}(S=2) \leftrightarrow{ }^{3} T_{1 g}(S=1)\right] .{ }^{25,26}$ According to the Tanabe-Sugano diagram for an octahedral $3 d^{4}$ ion, ${ }^{27}$ the HS-LS transition should occur for a CF of $10 D q$ $=27 B$, where $B$ is the Racah parameter of $\mathrm{MnF}_{6}^{3-}(B$ $=0.097 \mathrm{eV}) .{ }^{4}$ From this value and taking into account the equation of state of this $A \mathrm{MnF}_{4}(A=\mathrm{Na}, \mathrm{K}, \mathrm{Rb}, \mathrm{Cs})$ compound series ${ }^{23,24}$ and on the assumption that $10 D q$ depends on the Mn-F distance as $10 D q=K R^{-5}$, such as was found for different transition-metal fluoride complexes, ${ }^{4,28-31}$ we estimate a HS-LS transition pressure of about $10 \mathrm{GPa}$ provided that the $\mathrm{MnF}_{6}^{3-}$ coordination geometry is octahedral. The $A \mathrm{MnF}_{4}$ series provides the shortest Mn-F distance and hence the highest $\mathrm{CF}^{4}$ thus favoring a HS-LS transition to occur at moderate high pressures. However, the JT effect preserves the crystal to undergo the HS-LS transition around the estimated pressure. As we will see later, the large JTinduced tetragonal splitting of the parent octahedral $\mathrm{Mn}^{3+}$ $e_{g}\left(3 z^{2}-r^{2}, x^{2}-y^{2}\right)$ electron levels into $b_{1 g}+a_{1 g}$, increases the LS electron energy with respect to the HS state and hence the transition pressure required for electronic pairing (Fig. 1). Through this work, we estimate that the critical pressure required to induce a HS-LS transition is increased by the JT effect to about $150 \mathrm{GPa}$. Interestingly, $\mathrm{Mn}^{3+}$ compounds show also an intense pleochroism, which depends on both the JT distortion as well as the orientation of the $\mathrm{MnF}_{6}^{3-}$ complexes within the crystal. ${ }^{4,32}$ 

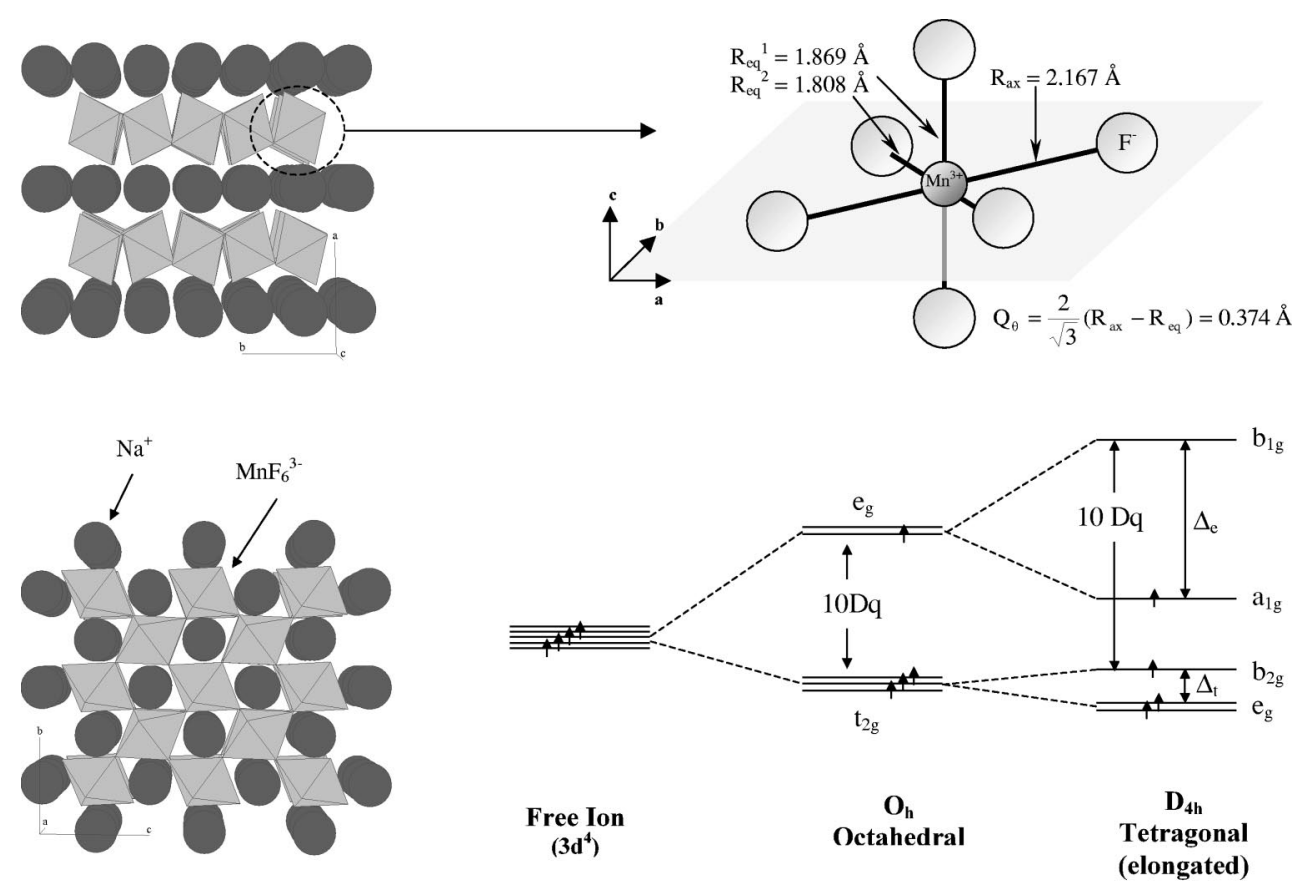

FIG. 1. Left: crystal structure of the $\mathrm{NaMnF}_{4}$-layered perovskite at ambient pressure. View of the $b$ - $c$ plane (top) and view of the layer along $c$ (bottom). Note the antiferrodistortive structure displayed by the corner-sharing $\mathrm{MnF}_{6}^{3-}$ octahedra. Right: (top) view of the nearly tetragonal elongated coordination of the $\mathrm{MnF}_{6}^{3-}$ complex, with the corresponding axial and equatorial Mn-F distances $R_{\mathrm{ax}}$ and $R_{\text {eq }}$, respectively. (bottom) Correlation diagram of the $d$ levels of $\mathrm{Mn}^{3+}$ in different symmetries.

This work investigates the $\mathrm{CF}$ electronic structure of $\mathrm{MnF}_{6}^{3-}$ in $\mathrm{NaMnF}_{4}$ and its variation with pressure in the 0-10 GPa range through optical absorption (OA) spectroscopy. The aim is to explore how the JT energy of $\mathrm{Mn}^{3+}$ and its associated coordination structure vary with pressure. These systems are attractive for this purpose since a rich band structure associated with the spin-allowed ${ }^{5} B_{1 g} \rightarrow{ }^{5} \Gamma_{i}$ $\left(\Gamma_{i}=A_{1 g}, B_{2 g}\right.$, and $\left.E_{g}\right)$ and spin-forbidden ${ }^{5} B_{1 g} \rightarrow{ }^{3} B_{1 g}$ transitions are well resolved in the VIS-UV range, thus providing a direct determination of the $\mathrm{CF}$ electron structure and, consequently, of the JT energy of $\mathrm{MnF}_{6}^{3-}$. We have developed a perturbed-octahedron complex model in order to establish correlations between the variation of the CF energies and the local structure around $\mathrm{Mn}^{3+}$, which strongly depends on both the volume and JT distortion of $\mathrm{MnF}_{6}^{3-} \cdot 4,14,32$ Knowledge of these correlations is important to elucidate whether the application of pressure reduces the JT distortion, leading to partial disappearance of the in-plane antiferrodistortive structure, or whether it induces out-ofplane tilts of the $\mathrm{MnF}_{6}^{3-}$ octahedra (Fig. 1). Moreover, it provides information about local-structure changes around $\mathrm{Mn}^{3+}$ under pressure from OA spectroscopy. This aspect is relevant since such information could not be obtained from $\mathrm{x}$-ray diffraction $(\mathrm{XRD})$ in previous structural works under pressure performed on $\mathrm{Mn}^{3+}$ fluorides $\left(A \mathrm{MnF}_{4}\right){ }^{22,23} \mathrm{Be}-$ sides $\mathrm{XRD}$, local-structure variations induced by pressure are not easy to accomplish through extended x-ray-absorption fine structure (EXAFS) under pressure due to the strong x-ray absorption of diamonds at the Mn $K$ edge on dealing with a diamond anvil cell (DAC). ${ }^{34}$

Finally, the structural changes that we found from the pro- posed model are correlated with variations undergone by the exchange-induced ${ }^{5} B_{1 g} \rightarrow{ }^{3} B_{1 g}$ spin-flip transitions, whose intensity is very sensitive to the superexchange Mn-F-Mn angle between adjacent $\mathrm{MnF}_{6}^{3-}$ complexes within the layer.

\section{EXPERIMENT}

Single crystals of $\mathrm{NaMnF}_{4}$ were grown from cold solutions of $\mathrm{MnF}_{3}$ and $\mathrm{NaF}$ fluorides in hydrofluoric acid following a method reported elsewhere. ${ }^{35,36}$ This layer compound is monoclinic $\left(P 2{ }_{1} / c\right.$ space group, $\left.Z=2\right)$ with lattice parameters $a=5.736 \AA, b=4.892 \AA, c=5.748 \AA$, and $\beta=108.1^{\circ}$ at room temperature. ${ }^{22}$ The structure consists of layers of interconnected $\left[\mathrm{MnF}_{2} \mathrm{~F}_{4 / 4}\right]^{3-}$ corner-sharing octahedra separated by $\mathrm{Na}$ ions. The octahedra display a $D_{2 h}$ symmetry (nearly $D_{4 h}$ ) as a consequence of the JT distortion and crystal anisotropy. The in-plane equatorial F ligand of one Mn acts as axial ligand of the nearest $\mathrm{Mn}$, leading to the layered antiferrodistortive structure shown in Fig. 1. The axial and equatorial Mn-F distances are $R_{\text {ax }}=2.167 \AA$ and $R_{\text {eq }}=1.839 \AA$. The equatorial distance actually corresponds to the average of the two equatorial distances, $R_{\text {eq1 }}=1.808 \AA$ and $R_{\text {eq2 }}=1.869$ A.. ${ }^{22}$

The OA spectra under pressure were obtained using a specially designed spectrophotometer. The monochromatic light in the UV-VIS-IR range was obtained by means of a Spectra Pro-300i ARC Monochromator and suitable filters. The light was chopped and detected with a Hamamatsu R-928 Phototube and a SR 830 Lock-in amplifier. The experimental setup is described elsewhere. ${ }^{37}$ Pressure experiments were done in a DAC (Diamond Optics, Inc.) using single crystals of 

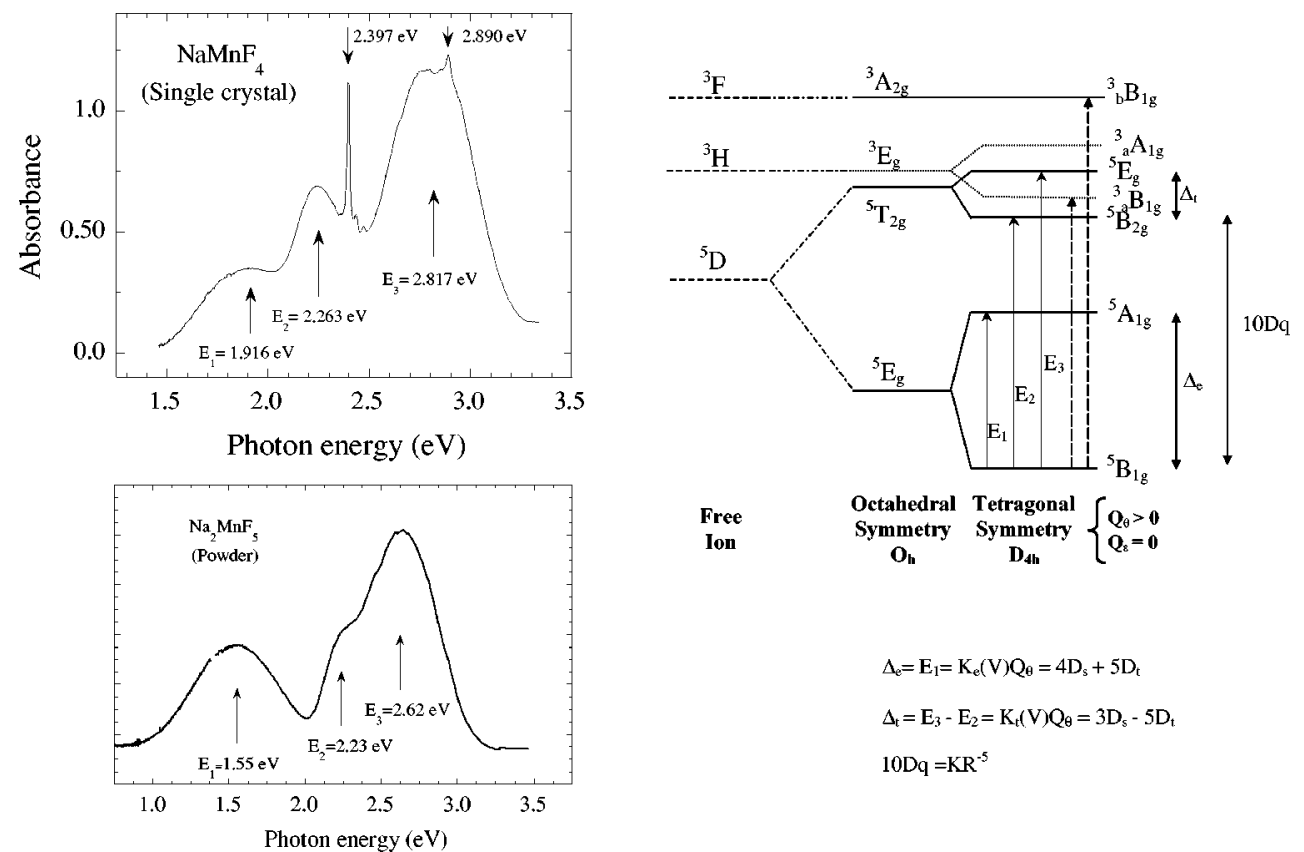

O

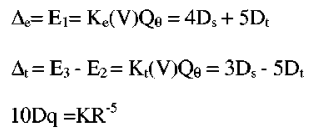

$10 \mathrm{Dq}=\mathrm{KR}^{-5}$

FIG. 2. Optical absorption spectra of $\mathrm{NaMnF}_{4}$ (single crystal) and $\mathrm{Na}_{2} \mathrm{MnF}_{5}$ (powder). An energy-state diagram of $\mathrm{Mn}^{3+}$ in elongated$D_{4 h}$ symmetry is also included. The assignment of the spin-allowed ${ }^{5} B_{1 g} \rightarrow{ }^{5} \Gamma_{i}\left(\Gamma_{i}=A_{1 g}, B_{2 g}\right.$, and $\left.E_{g}\right)$ bands and the spin-flip ${ }^{5} B_{1 g}$ $\rightarrow{ }^{3} B_{1 g}$ peaks is indicated by arrows. The expression for the tetragonal splitting of the $E_{g}\left(\Delta_{e}\right)$ and $T_{2 g}\left(\Delta_{t}\right)$ states as well as for $10 D q$ is given as a function of the normal coordinate $Q_{\theta}$ and $R_{\text {eq }}$, respectively. The expressions are also given as a function of the tetragonal CF parameters $D_{s}$ and $D_{t}$.

$\mathrm{NaMnF}_{4}\left(150 \times 120 \times 60 \mu \mathrm{m}^{3}\right)$. We used paraffin oil as pressure transmitting media in order to prevent oxidation. The pressure in the hydrostatic cavity was calibrated from the ruby $R$-line shift. The ruby was excited with the $530.9-\mathrm{nm}$ line of a $\mathrm{Kr}^{+}$ion laser (Coherent CR-500K).

\section{RESULTS AND DISCUSSION}

\section{A. Pressure spectroscopy on $\mathrm{NaMnF}_{4}$}

The CF spectrum of $\mathrm{Mn}^{3+}$ ions in the $A_{n} \mathrm{MnF}_{n+3}(A$ $=$ alkali ion, $n=1-3$ ) compound series can be understood on the basis of the JT-octahedral-distorted $\mathrm{MnF}_{6}^{3-}$ complex. ${ }^{4}$ Within an elongated $D_{4 h}$ coordination, the spectra of these compounds consist of three intense broadbands $E_{1}, E_{2}$, and $E_{3}$ (Fig. 2), which are resolved in the UV-VIS range. These bands are associated with electronic transitions ${ }^{5} B_{1 g} \rightarrow{ }^{5} \Gamma_{i}$ ( $\Gamma_{i}=A_{1 g}, B_{2 g}$, and $E_{g}$ ) involving states of the same spin, $S=2$, and their energy strongly depends on the CF. In addition, there are several narrow peaks corresponding to spinflip ${ }^{5} B_{1 g} \rightarrow{ }^{3} B_{1 g}$ transitions. These electronic transitions are electric-dipole (ED) forbidden since they involve different spin states. However, in these compounds they are activated by the exchange mechanism. ${ }^{4}$ In contrast to the spin-allowed ${ }^{5} B_{1 g} \rightarrow{ }^{5} \Gamma_{i}$ transitions, the spin-flip transitions are weakly $\mathrm{CF}$ dependent and thus they appear as narrow features in the OA spectrum.

Structural correlations performed on $\mathrm{Mn}^{3+}$ fluorides indicate that the tetragonal splitting associated with the parent octahedral $e_{g}$ and $t_{2 g}$ orbitals, termed $\Delta_{e}=E_{1}$ and $\Delta_{t}=E_{3}$ $-E_{2}$, respectively, is proportional to the JT distortion, which is characterized by the $Q_{\theta}$ normal coordinate in $\mathrm{MnF}_{6}^{3-}$ com- plexes with local $D_{2 h}$ symmetry (Fig. 1).,14,38 On the other hand, $E_{2}$ depends only on the equatorial Mn-F distance $R_{\text {eq }}$, and therefore its transition energy provides a very sensitive probe to detect variations of $R_{\text {eq }}$ upon pressure.

Figure 2 shows the OA spectrum of $\mathrm{NaMnF}_{4}$ at ambient conditions. The spectrum of the $1 \mathrm{D} \mathrm{Na} \mathrm{NnF}_{5}$ compound is also included for comparison purposes. The three $E_{1}, E_{2}$, and $E_{3}$ bands appear at 1.916, 2.263, and $2.817 \mathrm{eV}$, respectively, for $\mathrm{NaMnF}_{4}$. Note that these energies shift to lower energies in the less JT-distorted $\mathrm{MnF}_{6}^{3-}$ complex in $\mathrm{Na}_{2} \mathrm{MnF}_{5}$. The $\mathrm{CF}$ energies as well as the corresponding JT splitting, $\Delta_{e}=1.916 \mathrm{eV}$ and $\Delta_{t}=0.554 \mathrm{eV}$, reflect the nearly $D_{4 h}$ JT distortion of the $\mathrm{MnF}_{6}^{3-}$ characteristic of a 2D layered perovskite $A \mathrm{MnF}_{4}(A=\mathrm{K}, \mathrm{Rb}, \mathrm{Cs}, \mathrm{Tl})$ with $Q_{\theta}$ $=0.373 \AA$ and $Q_{\epsilon}=0.061 \AA$ for $\mathrm{NaMnF}_{4}{ }^{22}$ The $O_{h}$ normal coordinates $e_{g}\left(Q_{\theta}, Q_{\epsilon}\right)$ are derived from the equatorial and axial distances obtained from XRD through Eq. (1):

$$
Q_{\theta}=\frac{2}{\sqrt{3}}\left(R_{\mathrm{ax}}-R_{\mathrm{eq}}\right), \quad Q_{\epsilon}=R_{\mathrm{eq} 2}-R_{\mathrm{eq} 1},
$$

with $R_{\text {eq }}=\frac{1}{2}\left(R_{\text {eq } 1}+R_{\text {eq } 2}\right)$. Interestingly, these coordinates are useful when dealing with the $e \otimes E \mathrm{JT}$ effect in $O_{h}$ systems as well as with structural distortions induced by either hydrostatic pressure or axial stress in the framework of an $O_{h}$ perturbed complex. ${ }^{32}$

The influence of the $\mathrm{MnF}_{6}^{3-}$ distortion on the electron structure is clearly evidenced through the OA spectrum of the $1 \mathrm{D} \mathrm{Na}_{2} \mathrm{MnF}_{5}$ (Fig. 2). Its tetragonal splitting $\Delta_{e}=1.55$ $\mathrm{eV}$ and $\Delta_{t}=0.39 \mathrm{eV}$ reflects the smaller JT distortion: $Q_{\theta}$ $=0.34 \AA$ and $Q_{\epsilon}=0.03 \AA$, derived from XRD. ${ }^{39}$ The same 


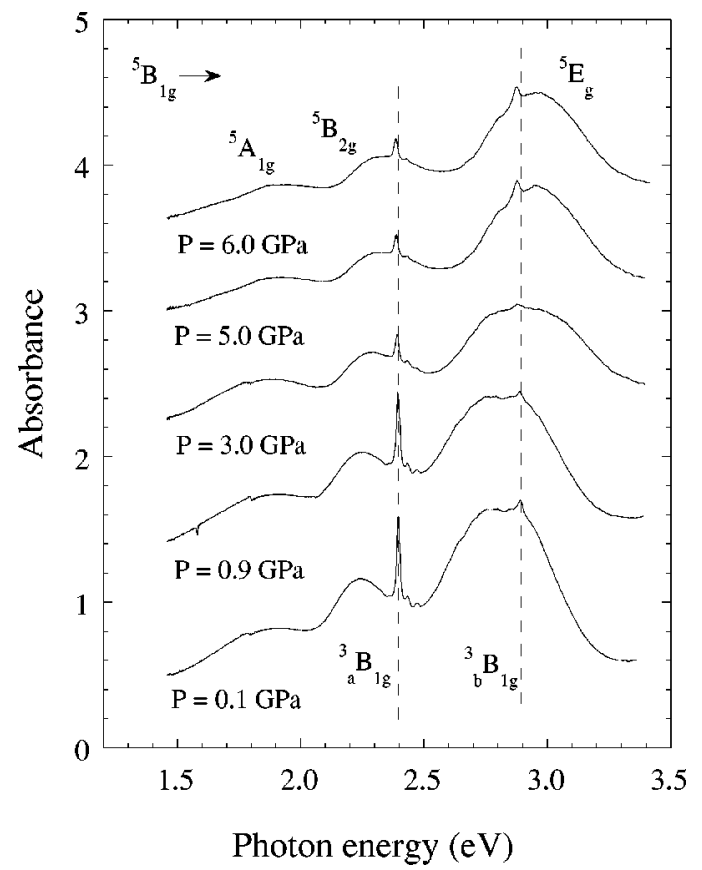

FIG. 3. Optical absorption spectrum of the $\mathrm{NaMnF}_{4}$ single crystal and its variation with the pressure. The peak assignment within a $D_{4 h}$ symmetry is indicated. Vertical dashed lines denote the position of the triplet peaks at ambient pressure.

trend is found on comparing the spectroscopic and structural parameters for the $0 \mathrm{D} \mathrm{Na} \mathrm{NnF}_{6}$ compound. The parameters are $\Delta_{e}=1.04 \mathrm{eV}$ and $\Delta_{t}=0.20 \mathrm{eV}$, which corresponds to a $\mathrm{MnF}_{6}^{3-}$ distortion: $Q_{\theta}=0.16 \AA$ and $Q_{\epsilon}=0.035 \AA{ }^{40,41}$ From these values we can directly obtain the JT energy at ambient conditions since $E_{1}=\Delta_{e}=4 E_{\mathrm{JT}}$. Therefore $E_{\mathrm{JT}}$ changes along the $\mathrm{Na}_{n} \mathrm{MnF}_{n+3}$ series as $0.48,0.39$, and $0.26 \mathrm{eV}$ for $n=1,2$, and 3, respectively. It is worth pointing out that $E_{\mathrm{JT}}$ and the corresponding complex distortion increase with the crystal dimensionality, i.e., with the number of shared ligands. This structural correlation does not only hold for $\mathrm{Na}$ compounds, but for the whole compound series, as was reported elsewhere. ${ }^{4}$

Figure 3 shows the variation of the OA spectrum with the pressure in $\mathrm{NaMnF}_{4}$. The effect of applying pressure is mainly to shift the three intense bands towards higher energies, whereas a slight redshift is observed for the two spinflip ${ }^{5} B_{1 g} \rightarrow{ }^{3} B_{1 g}$ peaks. The variation of $E_{1}, E_{2}$, and $E_{3}$ shows a linear behavior with the pressure. The corresponding coefficients are given in Fig. 4. The small pressure-induced shift of $E_{1}$ is worthwhile. It clearly indicates that $E_{\mathrm{JT}}$ does not change significantly with pressure. A slight increase of $\partial E_{\mathrm{JT}} / \partial P=0.8 \mathrm{meV} / \mathrm{GPa}$ is derived from $E_{1}(P)$ in Fig. 4. However, this result contrasts with the important increase experienced by the $t_{2 g}$ splitting, $\partial \Delta_{t} / \partial P=16 \mathrm{meV} / \mathrm{GPa}$, in comparison to $\partial \Delta_{e} / \partial P=3 \mathrm{meV} / \mathrm{GPa}$, in spite of $\Delta_{e}$ being larger than $\Delta_{t}$ at ambient conditions: $\Delta_{e}=3.5 \Delta_{t} \cdot{ }^{4}$ This puzzling feature is consequence of the different variations of the linear electron-lattice coupling coefficient for the oneelectron $e_{g}$ and $t_{2 g}$ levels ( $O_{h}$ scheme), aside from the structural changes experienced by the $\mathrm{MnF}_{6}^{4-}$ complex under pressure. Similar findings were also observed in the variation
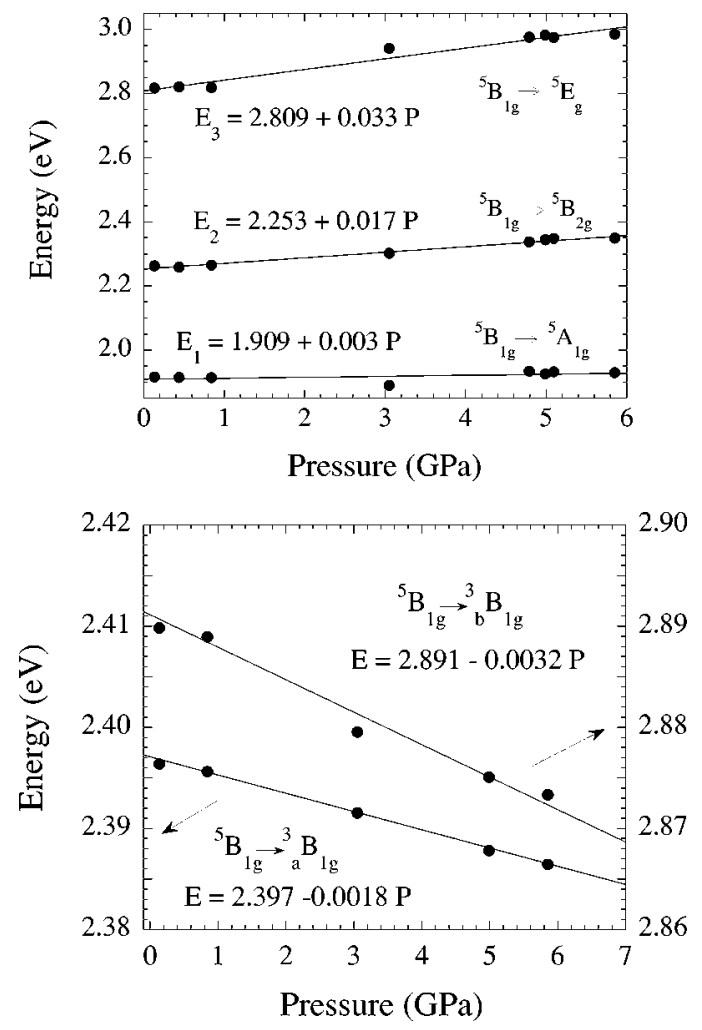

FIG. 4. Variation of the transition energy for the three intense bands $E_{1}, E_{2}$, and $E_{3}$ (top) and the narrow ${ }^{5} B_{1 g} \rightarrow{ }^{3} B_{1 g}$ peaks (bottom) with pressure. Straight lines and corresponding equations are the linear least-squares fits to the experimental data. The energy corresponds to the band maximum. The absolute errors for the energy obtained either from the band maximum or by fitting to gaussians is $10 \mathrm{meV}$, while the error of the energy variation with pressure is $3 \mathrm{meV}$ for $E_{1}, E_{2}$, and $E_{3}$. The corresponding errors for the triplets are 1 and $0.2 \mathrm{meV}$, respectively. The point size in both plots is slightly bigger than the actual pressure and energy errors. The fitting errors for $E$ and $\partial E / \partial P$ are $9 \mathrm{mev}$ and $2 \mathrm{meV} \mathrm{GPa}^{-1}$ for ${ }^{5} B_{1 g} \rightarrow{ }^{5} A_{1 g}$, respectively, $3 \mathrm{meV}$ and $1 \mathrm{meV} \mathrm{GPa}^{-1}$ for ${ }^{5} B_{1 g}$ $\rightarrow{ }^{5} B_{2 g}$, and $10 \mathrm{meV}$ and $3 \mathrm{meV} \mathrm{GPa}^{-1}$ for ${ }^{5} B_{1 g} \rightarrow{ }^{5} E_{g}$. For the triplets, the fitting errors are $0.4 \mathrm{meV}$ and $0.1 \mathrm{meV} \mathrm{GPa}^{-1}$ for ${ }^{5} B_{1 g} \rightarrow{ }_{3}^{a} B_{1 g}$ and $0.8 \mathrm{meV}$ and $0.2 \mathrm{meV} \mathrm{GPa}^{-1}$ for ${ }^{5} B_{1 g} \rightarrow{ }_{3}^{b} B_{1 g}$, respectively. The calculated energy shifts using the developed model coincide with fit lines. Note the different energy scale employed in each representation.

of the wave function coefficients $N_{e}$ and $N_{t}$ associated with the mainly metallic molecular orbitals $e_{g}$ and $t_{2 g}$ of $\mathrm{MnCl}_{6}^{4-}$ in pressure experiments carried out in $\mathrm{NH}_{4} \mathrm{MnCl}_{3}$. ${ }^{42}$ The pressure variation of $N_{e}$ is slightly smaller than the variation of $N_{t}$ in spite of $N_{e}>N_{t}$.

\section{B. Structural correlations: Octahedron-perturbed model}

From the present results, we are able to extract valuable information on the local structural changes around $\mathrm{Mn}^{3+}$ under pressure, provided that we know how $E_{1}, E_{2}$, and $E_{3}$ depend on $R_{\text {eq }}$ and $R_{\text {ax }}$ (or $R_{\text {eq }}$ and $Q_{\theta}$ ). The use of the $Q_{\theta}$ normal coordinate is important since the JT-tetragonal splittings $\Delta_{e}$ and $\Delta_{t}$ within a $D_{4 h}$ complex framework are both proportional to $Q_{\theta}$ for small deviations of the $O_{h}$ symmetry: $4,32,38$ 


$$
\Delta_{e}=K_{e}(V) Q_{\theta}, \quad \Delta_{t}=K_{t}(V) Q_{\theta} .
$$

Here $K_{e}$ and $K_{t}$ are the JT electron-lattice coupling coefficients that, in general, depend on the volume per molecule, $V$, or the pressure $P$. It must be remarked that the structural correlation reported along a series of $\mathrm{Mn}^{3+}$ fluorides ${ }^{4,43}$ indicates that $\Delta_{e}=5.08 Q_{\theta}$ and $\Delta_{t}=1.34 Q_{\theta}$ (units in $\mathrm{eV}$ and $\AA$ ), with a ratio $\Delta_{e} / \Delta_{t} \approx 4$ at ambient conditions. Note that the measured $\Delta_{e}$ and $\Delta_{t}$ values in $\mathrm{NaMnF}_{4}$ are in good agreement with this figure. We assume a simple $D_{4 h}$ symmetry as appropriate for the model analysis, since the occurrence of orthorhombic CF components is unlike as is evidenced from the lack of splitting associated the high-energy ${ }^{5} B_{1 g} \rightarrow{ }^{5} E_{g}$ transition (Fig. 3).

Interestingly, $E_{2}\left({ }^{5} B_{1 g} \rightarrow{ }^{5} B_{2 g}\right)$ only depends on the equatorial $\mathrm{Mn}-\mathrm{F}$ distance $R_{\text {eq }} \cdot{ }^{38}$ According to $\mathrm{CF}$ theory, its variation can be expressed as $E_{2}=K R_{\text {eq }}^{-n}$ with $n=5$. Values of $n$ between 4 and 5 have been obtained from calculations for trivalent ions $\mathrm{Cr}^{3+},{ }^{30,44} \mathrm{Fe}^{3+},{ }^{45}$ and spectroscopy. ${ }^{4,28,29}$ Assuming an average value of the exponent, $n=4.5$, we can easily derive the variation of $R_{\text {eq }}$ under pressure from the $E_{2}$ shift. It must be emphasized that the present method provides suitable variations of $R_{\text {eq }}$ irrespective of particular $n$ choice either 4 or 5 .

From the band shifts of Figs. 3 and 4, we conclude the following:

(i) The equatorial Mn-F distance $R_{\text {eq }}$ decreases with increasing pressure as is evidenced by the blueshift: $\partial E_{2} / \partial P$ $=17 \mathrm{meV} / \mathrm{GPa}$.

(ii) The variation of $R_{\text {eq }}$ with pressure can be obtained from the pressure derivatives of $E_{2}$ :

$$
\frac{\partial E_{2}}{\partial P}=\frac{\partial E_{2}}{\partial R_{\mathrm{eq}}} \frac{\partial R_{\mathrm{eq}}}{\partial P},
$$

with

$$
\frac{\partial E_{2}}{\partial R_{\text {eq }}}=-n \frac{E_{2}}{R_{\text {eq }}}=-4.5 \frac{2.263}{1.84}=-5.53 \mathrm{eV} / \AA \text {, }
$$

so that we get $\partial R_{\text {eq }} / \partial P=-3.1 \times 10^{-3} \AA / G P a$, which means a variation of $\Delta R_{\mathrm{eq}}=-0.031 \AA$ from ambient pressure to 10 GPa.

(iii) The present spectroscopic procedure provides a suitable method for deriving bond-distance variations that actually improve the x-ray absorption spectroscopy (XAS) sensitivity. By using Eq. (3), an energy-shift accuracy of $5 \mathrm{meV}$ leads to a bond-distance accuracy of $10^{-3} \AA$.

(iv) The $\mathrm{MnF}_{6}^{3-}$ JT distortion does not change significantly upon pressure. Note that disappearance of the JT effect yielding $Q_{\theta} \approx 0$ should induce splitting closure of the ${ }^{5} E_{g}$ and ${ }^{5} T_{2 g}$ states: $\Delta_{e}=0$ and $\Delta_{t}=0$ (Fig. 1). On the contrary, we observe that both $\Delta_{e}$ and $\Delta_{t}$ increase by 0.032 and $0.161 \mathrm{eV}$, respectively, from ambient pressure to $10 \mathrm{GPa}$. Moreover, the variation of $\Delta_{t}$ in the same pressure range is 5 times the variation of $\Delta_{e}$ (Fig. 4). (v) The fact that $\partial \Delta_{e}(P) / \partial P=3 \mathrm{meV} / \mathrm{GPa}$ and $\partial \Delta_{t}(P) / \partial P=16 \mathrm{meV} / \mathrm{GPa}$ implies necessarily that $K_{e}$ and $K_{t}$ must both increase with pressure and $\Delta K_{e} / K_{e}$ $\ll \Delta K_{t} / K_{t}$.

The relation between the electron-lattice coupling parameters and the crystal volume can be expressed for small departures of the $O_{h}$ symmetry as follows: ${ }^{37,38}$

$$
K_{e}(V)=K_{e}^{0}\left(\frac{V_{0}}{V}\right)^{n_{e}}, \quad K_{t}(V)=K_{t}^{0}\left(\frac{V_{0}}{V}\right)^{n_{t}} .
$$

The electron-lattice coupling and the crystal volume parameters at ambient conditions for $\mathrm{NaMnF}_{4}$ are $K_{e}^{0}=5.05 \mathrm{eV} / \AA$, $K_{t}^{0}=1.46 \mathrm{eV} / \AA$, and $V_{0}=153.3 \AA^{3}$. The results of Figs. 3 and 4 can be explained in the framework of the JT model using effective values $n_{e}=0.7$ and $n_{t}=2.2$. These exponents are similar to those from $\mathrm{CF}$ theory ${ }^{38}$ and also from extended-Hückel and $X \alpha$ complex calculations ${ }^{46,47}$ yielding values of $n_{e}$ and $n_{t}$ around 1 and 2, respectively. However, it must be remarked that the particular choice of $n_{e}$ and $n_{t}$ around those values does not affect significantly, as we show later, the quantitative estimates performed on this work. The selected values provide similar values of the pressure derivatives of the JT electron-lattice coupling parameter, $\partial K_{e} / \partial P$ $\approx \partial K_{t} / \partial P$, in agreement with previous studies on the variation of the wave function coefficients $N_{t}$ and $N_{e}$ associated with the $e_{g}$ and $t_{2 g}$ orbitals with pressure $\partial N_{e} / \partial P$ $\approx \partial N_{t} / \partial P .^{9}$

The structural constraint inferred from Eq. (2) requires that $\Delta_{e}(P) / K_{e}(P)=\Delta_{t}(P) / K_{t}(P)$ for any tetragonal perturbation $Q_{\theta}$. It means that, for small departures from $O_{h}$ symmetry, the splitting and associated electron-lattice coupling coefficient of $e_{g}$ and $t_{2 g}$ are related by the equation

$$
\frac{1}{K_{e}^{0}} \frac{\partial K_{e}}{\partial P}-\frac{1}{\Delta_{e}^{0}} \frac{\partial \Delta_{e}}{\partial P}=\frac{1}{K_{t}^{0}} \frac{\partial K_{t}}{\partial P}-\frac{1}{\Delta_{t}^{0}} \frac{\partial \Delta_{t}}{\partial P},
$$

and according to Eq. (4), the pressure derivatives of $K_{e}$ and $K_{t}$ are given by

$$
\frac{1}{K_{e}^{0}} \frac{\partial K_{e}}{\partial P} \approx-n_{e} \frac{1}{V_{0}} \frac{\partial V}{\partial P}, \quad \frac{1}{K_{t}^{0}} \frac{\partial K_{t}}{\partial P} \approx-n_{t} \frac{1}{V_{0}} \frac{\partial V}{\partial P},
$$

and therefore,

$$
\frac{1}{K_{t}^{0}} \frac{\partial K_{t}}{\partial P}-\frac{1}{K_{e}^{0}} \frac{\partial K_{e}}{\partial P} \approx\left(n_{e}-n_{t}\right) \frac{1}{V_{0}} \frac{\partial V}{\partial P} \approx \frac{\left(n_{e}-n_{t}\right)}{B_{0}} .
$$

Taking a bulk modulus $B_{0}=60 \mathrm{GPa}$ (Refs. 5 and 23) and combining Eqs. (5) and (7) with the experimental data of Fig. 4, we get $n_{t}-n_{e}=1.6$. According to Eq. (6), the ratio

$$
\frac{n_{t}}{n_{e}}=\frac{K_{e}^{0}}{K_{t}^{0}} \frac{\partial K_{t} / \partial P}{\partial K_{e} / \partial P}=3.4,
$$

on the assumption that $\partial K_{e} / \partial P \approx \partial K_{t} / \partial P$. Hence we obtain $n_{t}=2.2$ and $n_{e}=0.7$, so that we get $\partial K_{e} / \partial P \approx \partial K_{t} / \partial P=55$ meV/Å GPa, which means an electron-lattice coupling at 10 $\mathrm{GPa}$ of $K_{e}(10)=5.60 \mathrm{eV} / \AA$ and $K_{t}(10)=2.01 \mathrm{eV} / \AA$. 
Consequently, the $D_{4 h} \mathrm{JT}$ distortion of $\mathrm{MnF}_{6}^{3-}$ in $\mathrm{NaMnF}_{4}$ at this pressure is

$$
Q_{\theta}=\frac{\Delta_{e}(10)}{K_{e}(10)}=\frac{1.91}{5.60} \approx \frac{\Delta_{t}(10)}{K_{t}(10)}=\frac{0.71}{2.01} \approx 0.345 \AA .
$$

In conclusion, the application of pressure induces a reduction of the tetragonal distortion from $0.379 \AA$ at ambient pressure to $0.345 \AA$ at $10 \mathrm{GPa}: \Delta Q_{\theta}=-0.034 \AA$. In terms of bond distances, it means a reduction of $R_{\text {eq }}$ and $R_{\mathrm{ax}}$ from 1.839 to $1.808 \AA$ for $R_{\text {eq }}$ and from 2.167 to $2.107 \AA$ for $R_{\mathrm{ax}}$, e.g., $\Delta R_{\text {eq }}=-0.031 \AA$ and $\Delta R_{\mathrm{ax}}=-0.060 \AA$ in the $0-10 \mathrm{GPa}$ range (Table I).

\section{Structural correlations based on the crystal-field theory}

A similar structural correlation is obtained on the basis of the CF theory (or ligand-field theory following Griffith's criterion). ${ }^{48}$ Within this theory the tetragonal splitting for the $e_{g}$ and $t_{2 g}$ levels is given by $\Delta_{e}=4 D_{s}+5 D_{t}$ and $\Delta_{t}=3 D_{s}$ $-5 D_{t} \cdot{ }^{4,38}$ The advantage of using parameters $D_{s}$ and $D_{t}$ lies on the fact that they depend explicitly on the Mn-F bond distances as ${ }^{38}$

$$
\begin{aligned}
& D_{s}=\frac{2}{7} e^{2}\left\langle r^{2}\right\rangle_{3 d}\left(\frac{1}{R_{\mathrm{eq}}^{3}}-\frac{1}{R_{\mathrm{ax}}^{3}}\right), \\
& D_{t}=\frac{2}{21} e^{2}\left\langle r^{4}\right\rangle_{3 d}\left(\frac{1}{R_{\mathrm{eq}}^{5}}-\frac{1}{R_{\mathrm{ax}}^{5}}\right) .
\end{aligned}
$$

Taking the electron matrix elements as semiempirical parameters, analogously to what is usually done on dealing with the CF parameter $10 D q$, the dependence of $D_{s}$ and $D_{t}$ on small tetragonal distortions from the $O_{h}$ symmetry is given by

$$
D_{s}=C_{s} \frac{Q_{\theta}}{R_{\mathrm{eq}}^{4}}, \quad D_{t}=C_{t} \frac{Q_{\theta}}{R_{\mathrm{eq}}^{6}} .
$$

Parameters $C_{s}$ and $C_{t}$ can be empirically determined from structural and spectroscopic data at ambient conditions. According to expressions given in Fig. 4, the pressure dependence of $D_{s}$ and $D_{t}$ is

$$
\begin{gathered}
D_{s}=\frac{1}{7}\left(\Delta_{e}+\Delta_{t}\right)=0.352+0.0027 P, \\
D_{t}=\frac{3}{35} \Delta_{e}-\frac{4}{35} \Delta_{t}=0.100-0.0016 P .
\end{gathered}
$$

On differentiating Eq. (9) with respect to $P$, we obtain

$$
\begin{gathered}
\frac{1}{D_{s}} \frac{\partial D_{s}}{\partial P}=\frac{1}{Q_{\theta}} \frac{\partial Q_{\theta}}{\partial P}-\frac{4}{R_{\mathrm{eq}}} \frac{\partial R_{\mathrm{eq}}}{\partial P}, \\
\frac{1}{D_{t}} \frac{\partial D_{t}}{\partial P}=\frac{1}{Q_{\theta}} \frac{\partial Q_{\theta}}{\partial P}-\frac{6}{R_{\mathrm{eq}}} \frac{\partial R_{\mathrm{eq}}}{\partial P} .
\end{gathered}
$$

TABLE I. Relevant structural and spectroscopic parameters for $\mathrm{NaMnF}_{4}$ at 0 and $10 \mathrm{GPa}$. Here $E_{1}, E_{2}$, and $E_{3}$ are the CF energies associated with transitions between one-electron orbitals $b_{1 g}\left(x^{2}\right.$ $\left.-y^{2}\right) \rightarrow a_{1 g}\left(3 z^{2}-r^{2}\right), \quad b_{1 g}\left(x^{2}-y^{2}\right) \rightarrow b_{2 g}(x y), \quad$ and $b_{1 g}\left(x^{2}-y^{2}\right)$ $\rightarrow e_{g}(x z, y z)$, respectively. Parameters $R_{\text {eq }}$ and $R_{\text {ax }}$ are the equatorial and axial $\mathrm{Mn}-\mathrm{F}$ distances of the $\mathrm{MnF}_{6}^{3-}$ complex. The tetragonal normal coordinate $Q_{\theta}$ is related to these distances as $Q_{\theta}=(2 / \sqrt{3})$ $\times\left(R_{\mathrm{ax}}-R_{\mathrm{eq}}\right)$. Structural data at $P=10 \mathrm{GPa}$ were derived from the structural correlation established in Sec. III B.

\begin{tabular}{lccc}
\hline \hline & $P=0$ & $P=10 \mathrm{GPa}$ & $\Delta E$ or $\Delta R$ \\
\hline$\Delta_{e}=E_{1}(\mathrm{eV})$ & 1.909 & 1.941 & 0.032 \\
$\Delta_{t}=E_{3}-E_{2}(\mathrm{eV})$ & 0.556 & 0.716 & 0.16 \\
$10 D q=E_{2}(\mathrm{eV})$ & 2.253 & 2.423 & 0.17 \\
$R_{\mathrm{eq}}(\AA)$ & 1.839 & 1.808 & -0.031 \\
$R_{\mathrm{ax}}(\AA)$ & 2.167 & 2.107 & -0.060 \\
$Q_{\theta}(\AA)$ & 0.379 & 0.345 & -0.034 \\
\hline \hline
\end{tabular}

Equation (11) provides the pressure derivative of the normal coordinate $Q_{\theta}$ using Eq. (10) and the pressure derivative of $R_{\text {eq }}$ from Eq. (3). We obtain an average value

$$
\frac{1}{Q_{\theta}} \frac{\partial Q_{\theta}}{\partial P}=-0.009(3) \mathrm{GPa}^{-1} \text {. }
$$

It means that $Q_{\theta}$ decreases with pressure by $\Delta Q_{\theta}=$ $-0.034(11) \AA$ from 0 to $10 \mathrm{GPa}$, which is similar to the previous finding. Table I summarizes the local structural changes undergone by the $\mathrm{MnF}_{6}^{3-}$ with pressure following the structural correlations established in this work.

In conclusion, the effect of pressure is mainly to reduce all $\mathrm{Mn}-\mathrm{F}$ distances of the $\mathrm{MnF}_{6}^{3-}$ complex. The axial distance reduces twice the equatorial distance, thus leading to a partial reduction of the JT distortion. This scenario agrees with previous findings in the antiferrodistortive $\left[\mathrm{C}_{3} \mathrm{H}_{7} \mathrm{NH}_{3}\right]_{2} \mathrm{CuCl}_{4}$ crystal using XRD and EXAFS under pressure. ${ }^{32,49}$ A similar result was also reported for $\mathrm{LaMnO}_{3}$ using Raman spectroscopy and XRD under pressure, ${ }^{12}$ although the observed JT reduction with pressure was not detected so far from neutron diffraction under pressure. ${ }^{50}$

As a remarkable feature, we have demonstrated the increase of the electron-lattice coupling related to the JT effect with pressure. Particularly, the relative pressure variation of $K_{t}$ is greater than that of $K_{e}$. Finally, it must be noted that from the proposed structural variation, the $\mathrm{MnF}_{6}^{3-}$ complex leads to a local bulk modulus $B_{\text {local }}=161 \mathrm{GPa}$, and therefore the complex appears to be less compressible than the crystal. This feature likely indicates the existence of $\mathrm{MnF}_{6}^{3-}$ tilts upon pressure, in agreement with previous findings in the perovskite layers $A_{2} \mathrm{CuCl}_{4}$ (Refs. 32 and 49) under pressure and along the $A \mathrm{MnF}_{4}$ series. ${ }^{4,14}$

\section{Exchange effects on the spin-flip ${ }^{5} B_{1 g} \rightarrow{ }^{3} B_{1 g}$ transition}

The pressure dependence of the peak intensity associated the spin-flip ${ }^{5} B_{1 g} \rightarrow{ }^{3} B_{1 g}$ transition at $2.397 \mathrm{eV}$ supports the proposed structural changes. Figure 5 shows the variation of the integrated peak intensity as a function of pressure. 


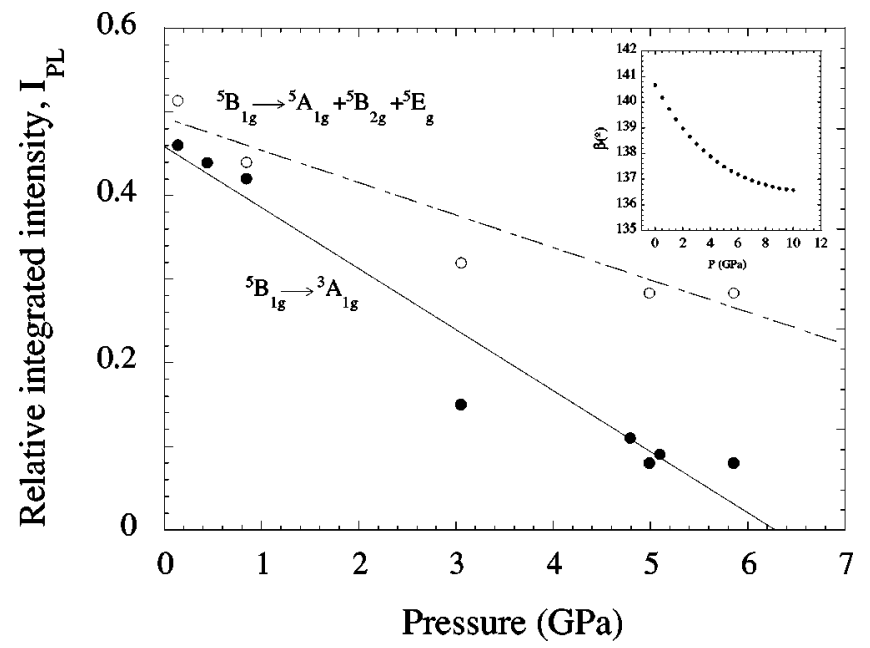

FIG. 5. Variation of the relative integrated intensity of the first ${ }^{5} B_{1 g} \rightarrow{ }^{3} B_{1 g}$ peak and the sum of the three band intensities ${ }^{5} B_{1 g}$ $\rightarrow{ }^{5} A_{1 g},{ }^{5} B_{1 g} \rightarrow{ }^{5} B_{2 g}$, and ${ }^{5} B_{1 g} \rightarrow{ }^{5} E_{g}$ with pressure. The inset shows the calculated superexchange F-Mn-F angle $\beta$ as a function of pressure. The angle was derived from structural correlations established in this work (see text).

Whereas the intensity of the spin-allowed CF bands decreases with pressure by about $40 \%-50 \%$, it falls a factor of 6 from 0 to $6 \mathrm{GPa}$ for the exchange-induced ${ }^{5} B_{1 g} \rightarrow{ }^{3} B_{1 g}$ transition. The intensity reduction is noteworthy since it reflects the proposed structural change with pressure. We associate this phenomenon with a decrease of the superexchange angle $(\beta)$ between $\mathrm{Mn}^{3+}$ neighbors due to pressure-induced tilting of the $\mathrm{MnF}_{6}^{3-}$ octahedra. The exchange-induced ED mechanism is known to be very sensitive to $\beta,{ }^{4}$ in such a way that deviations from $\beta=180^{\circ}$ yield a reduction of the associated transition oscillator strength. Besides the magnetostructural correlation established between the exchange constant $J$ measured along the $1 \mathrm{D} \mathrm{Mn}^{3+}$ compound series by magnetic techniques and the Mn-F-Mn tilting angle $\beta$ derived from XRD (Ref. 8) indicates that $J$ decreases with $\beta$. This correlation is consistent with present spectroscopic findings if we assume that the exchange-induced ED is somehow proportional to $J$. Figure 5 compares the OA data for ${ }^{5} B_{1 g}$ $\rightarrow{ }^{3} B_{1 g}$ with the variation of $\beta$ derived from pressure experiments using the local-structure data given in Table I and the equation of state for this compound series. ${ }^{5,23}$ Although the so-obtained $\beta$ values are derived on the assumption of an ideal perovskite layer structure, given the absence of suitable structural data for this compound, ${ }^{5,23,33}$ the variation of $\beta$ with pressure is consistent with XRD data along the $A \mathrm{MnF}_{4}$ series. In fact, the variation of $\Delta \beta=-5^{\circ}$ on passing from $\mathrm{KMnF}_{4}$ to $\mathrm{NaMnF}_{4}$ is associated with a volume contraction of $\Delta V / V_{0}=-11 \% .^{5,6}$ A similar situation is encountered for $\mathrm{NaMnF}_{4}$ upon hydrostatic pressure. The volume contraction of $\Delta V / V_{0}=-10 \%$ from ambient pressure to $10 \mathrm{GPa}$ is accompanied by an increase of the tilting angle; i.e., $\beta$ varies from $141^{\circ}$ to $137^{\circ}$ in the $0-10 \mathrm{GPa}$ range (inset of Fig. 5). Therefore the present results support the proposed structure variation with pressure as well as the occurrence of tilts as the main cause for intensity reduction. A project to perform suitable XRD experiments under pressure in $\mathrm{NaMnF}_{4}$ to confirm the proposed model is currently in progress.

\section{CONCLUSIONS}

We have investigated the variation of the $\mathrm{CF}$ electron structure of $\mathrm{Mn}^{3+}$ in $\mathrm{NaMnF}_{4}$ under pressure. Additionally, we have developed a perturbative octahedron-complex model to establish correlations between the electron $\mathrm{CF}$ and the local structure around $\mathrm{Mn}^{3+}$. The pressure-induced structural variation derived from this model agrees with estimations based on the CF theory. The model is advantageous since the observed shift rates can be described in terms of linear electron-lattice coupling coefficients whose values are obtained from structural correlations. The main conclusions of this work are the following. (1) The JT energy does not change significantly, but slightly increases with pressure. (2) The observed variation is interpreted in terms of reduction of the JT distortion $\left(\Delta Q_{\theta}<0\right)$, accompanied by pressure enhancement of electron-lattice coupling coefficients $(e \otimes E \mathrm{JT}$ effect). The competition between these two opposite contributions determines the weak increase of JT energy with pressure. (3) The effect of pressure is mainly to reduce the Mn-F distances. $R_{\mathrm{ax}}$ decreases about twice the reduction of $R_{\mathrm{eq}}$, thus leading to a partial reduction of the JT distortion and tilts of the $\mathrm{MnF}_{6}^{3-}$ octahedra. (4) We associate the strong decrease of the exchange-dependent ${ }^{5} B_{1 g} \rightarrow{ }^{3} B_{1 g}$ peak intensity with the occurrence of pressure-induced tilts. They reduce the superexchange interaction responsible for the ED transition mechanism and hence the intensity according to magnetostructural correlations established along the $\mathrm{Mn}^{3+}$ compound series.

\section{ACKNOWLEDGMENTS}

F.A. is indebted to the Ministerio de Ciencia y Tecnología (MCyT) for a FPI Research Grant (No. FP99, Project No. PB98-0190). This work was financed by the Spanish MCyT (Project No. BFM2001-0695) and the Vicerrectorado de Investigación of the University of Cantabria.
*Corresponding author. Electronic address: rodriguf@unican.es

${ }^{1}$ K. Petermann and G. Huber, J. Lumin. 31\&32, 71 (1984).

${ }^{2}$ S. Kück, S. Hartung, S. Hurling, K. Petermann, and G. Huber, Phys. Rev. B 57, 2203 (1998).

${ }^{3}$ S. Kück, S. Hartung, S. Hurling, and K. Petermann, Laser Phys. 8, 206 (1998).

${ }^{4}$ F. Rodríguez, P. Núñez, and M. C. Marco de Lucas, J. Solid State Chem. 110, 370 (1994).
${ }^{5}$ M. C. Morón, F. Palacio, S. M. Clark, and A. Paduan-Filho, Phys. Rev. B 51, 8660 (1995).

${ }^{6}$ F. Palacio and M. C. Morón, in Research Frontiers in Magnetochemistry, edited by C. J. O'Connor (World Scientific, Singapore, 1993).

${ }^{7}$ M. C. Morón, F. Palacio, and J. Rodriguez-Carvajal, J. Phys.: Condens. Matter 5, 4509 (1993).

${ }^{8}$ W. Massa and J. Steiner, J. Solid State Chem. 32, 137 (1980). 
${ }^{9}$ M. R. Ibarra, P. A. Algarabel, C. Marquina, J. Blasco, and J. García, Phys. Rev. Lett. 75, 3541 (1995).

${ }^{10}$ J. M. de Teresa, M. R. Ibarra, J. Blasco, J. García, C. Marquina, P. A. Algarabel, Z. Arnold, K. Kamenev, C. Ritter, and R. von Helmolt, Phys. Rev. B 54, 1187 (1996).

${ }^{11}$ J. Blasco, J. García, J. M. de Teresa, M. R. Ibarra, J. Perez, P. A. Algarabel, C. Marquina, and C. Ritter, Phys. Rev. B 55, 8905 (1997).

${ }^{12}$ I. Loa, P. Adler, A. Grzechnik, K. Syassen, U. Schwarz, M. Hanfland, G. Kh. Rozenberg, P. Gorodetsky, and M. P. Pasternak, Phys. Rev. Lett. 87, 125501 (2001).

${ }^{13}$ R. Dingle, Inorg. Chem. 4, 1287 (1965).

${ }^{14}$ D. Oeklrug, Struct. Bonding (Berlin) 9, 1 (1971).

${ }^{15}$ P. Kohler, W. Massa, D. Reinen, B. Hoffman, and R. Hoppe, Z. Anorg. Allg. Chem. 466, 131 (1978).

${ }^{16}$ A. Tressaud and J. M. Dance, Struct. Bonding (Berlin) 52, 87 (1982).

${ }^{17}$ W. Massa and D. Babel, Chem. Rev. (Washington, D.C.) 88, 275 (1988).

${ }^{18}$ P. Nuñez, A. Tressaud, J. Darriet, P. Hagenmuller, G. Hahn, G. Frenzen, W. Massa, D. Babel, A. Boireau, and J. L. Soubeyroux, Inorg. Chem. 31, 770 (1992).

${ }^{19}$ W. Massa, Inorg. Chem. 19, 117 (1999).

${ }^{20}$ P. Kohler, W. Massa, D. Reinen, B. Hofmann, and R. Hope, Z. Anorg. Allg. Chem. 446, 131 (1978).

${ }^{21}$ W. Massa, Acta Crystallog. Sect. C 42, 664 (1986).

${ }^{22}$ M. Molinier, W. Massa, S. Khairoun, A. Tressaud, and J. L. Soubeyroux, Z. Naturforsch. B 46, 1661 (1991).

${ }^{23}$ M. C. Morón, F. Palacio, and S. M. Clark, Phys. Rev. B 54, 7052 (1996).

${ }^{24}$ M. Molinier and W. Massa, Z. Naturforsch. B 47, 783 (1992).

${ }^{25}$ S. Carlson, Y. Xu, U. Halenius, and R. Norrestam, Inorg. Chem. 37, 1486 (1998).

${ }^{26}$ S. Carlson, Y. Xu, and R. Norrestam, Z. Kristallogr. 214, 269 (1999).

${ }^{27}$ Y. Tanabe and S. Sugano, J. Phys. Soc. Jpn. 9, 753 (1954).

${ }^{28}$ F. Rodríguez and M. Moreno, J. Chem. Phys. 84, 692 (1986).

${ }^{29}$ F. Rodriguez, M. Moreno, A. Tressaud, and J. P. Chaminade, Cryst. Lattice Defects Amorphous Mater. 16, 221 (1987).

${ }^{30}$ A. M. Woods, R. S. Sinkovits, and R. H. Bartram, J. Phys. Chem. Solids 55, 91 (1994).

${ }^{31}$ M. C. Marco de Lucas, F. Rodríguez, and M. Moreno, Phys. Rev.
B 50, 2760 (1994).

${ }^{32}$ R. Valiente and F. Rodríguez, Phys. Rev. B 60, 9423 (1999).

${ }^{33}$ S. Carlson and R. Norrestam, Z. Kristallogr. 210, 489 (1995); and (private communication).

${ }^{34} \mathrm{~J}$. P. Itiè (private communication).

${ }^{35}$ M. Molinier, W. Massa, S. Khairoun, A. Tressaud, and J. L. Soubeyroux, Z. Naturforsch. B 46, 1669 (1991).

${ }^{36}$ P. Nuñez, A. Tressaud, J. Grannec, P. Hagenmuller, W. Massa, D. Babel, A. Boireau, and J. L. Soubeyroux, Z. Anorg. Allg. Chem. 609, 71 (1992).

${ }^{37}$ F. Aguado, Graduate thesis, University of Cantabria, 2002.

${ }^{38}$ A. B. P. Lever, Inorganic Electronic Spectroscopy (Elsevier, Amsterdam, 1984).

${ }^{39}$ W. Massa, Acta Crystallogr., Sect. C: Cryst. Struct. Commun. 42, 664 (1986)

${ }^{40}$ U. Enlich, W. Massa, and T. Tresaud, Acta Crystallogr., Sect. C: Cryst. Struct. Commun. 48, 6 (1992).

${ }^{41}$ P. Kohler, W. Massa, D. Reinen, B. Hofmann, and R. Hope, Z. Anorg. Allg. Chem. 446, 131 (1978).

${ }^{42}$ D. Hernández, F. Rodríguez, M. Moreno, and H. U. Güdel, Physica B 265, 186 (1999).

${ }^{43}$ F. Aguado, F. Rodríguez, and P. Núñez, High Press. Res. 22, 121 (2002).

${ }^{44}$ M. Moreno, J. A. Aramburu, and M. T. Barriuso, Phys. Rev. B 56, 14423 (1997).

${ }^{45}$ J. A. Aramburu, J. I. Paredes, M. T. Barriuso, and M. Moreno, Phys. Rev. B 61, 6525 (2000).

${ }^{46}$ M. Moreno, M. T. Barriuso, and J. A. Aramburu, Int. J. Quantum Chem. 52, 829 (1994).

${ }^{47}$ M. T. Barriuso, J. A. Aramburu, M. Moreno, M. Flórez, G. Fernández Rodrigo, and L. Pueyo, Phys. Rev. B 38, 4239 (1988).

${ }^{48}$ J. S. Griffith, The Theory of Transition Metal Ions (Cambridge University Press, Cambridge, England, 1980), pp. 193-194.

${ }^{49}$ F. Rodríguez, M. Hanfland, J. P. Itiè, and A. Polian, in Frontiers of High Pressure Research II: Application of High Pressure to Low-Dimensional Novel Electronic Materials, edited by H. D. Hochheimer, B. Kuchta, P. K. Dorthout, and J. L. Yarger (Kluwer Academic, Dordrecht, 2001), Vol. 48, pp. 143-153.

${ }^{50}$ L. Pinsard-Gaudart, J. Rodríguez-Carvajal, A. Daoud-Aladine, I. Goncharenko, M. Medarde, R. I. Smith, and A. Revcolevschil, Phys. Rev. B 64, 064426 (2001). 